\title{
A Fuzzy Clustering Approach for Non-cooperative Behavior Detection in Consensus Reaching Processes
}

\author{
Iván Palomares ${ }^{1}$ Luis Martínez ${ }^{1}$ Francisco Herrera $^{2}$ \\ ${ }^{1}$ Computer Science Department. University of Jaén. Jaén 23071, Spain. \\ ${ }^{2}$ Computer Science and AI Department. University of Granada. Granada 18071, Spain. \\ \{ivanp, martin\}@ujaen.es, herrera@decsai.ugr.es
}

\begin{abstract}
Consensus reaching processes in group decision making attempt to reach a mutual agreement amongst experts before making a common decision. Classical consensus models are focused on problems where few decision makers participate. However, new societal and technological trends may require a large number of experts in such processes. In group decision making problems involving large groups, identifying and dealing with experts who present noncooperative behaviors during the consensus reaching process might become a particularly complex task. Such behaviors might bias the discussion process and prevent achieving an agreement. This paper presents a fuzzy clustering-based approach to detect and manage non-cooperative behaviors. Such an approach is integrated into a consensus model suitable to manage large groups of experts in group decision making problems.
\end{abstract}

Keywords: Group Decision Making, Consensus Reaching, Preference Relation, Fuzzy Clustering, Behavior Detection

\section{Introduction}

Decision making is a frequent mankind activity in daily life. Group Decision Making (GDM) problems, in which multiple experts with different points of view must make a common decision, have become increasingly necessary in many organizations [1].

Although GDM problems have been traditionally solved by applying a selection process to choose the best alternative/s [2], many real-life problems where entire groups are involved may require highly agreed collective decisions. Consensus Reaching Processes (CRPs) attempt to reach an agreement amongst experts before making a decision which is more accepted by the whole group [3].

Despite a variety of consensus models have been proposed to deal with CRPs [4-6], most of them focus on dealing with a few number of experts only. Current technological and societal demands are giving rise to new trends, such as e-democracy [7] and social networks [8], where decisions can be made by a large number of experts. In such situations, the CRP would present a higher cost and complexity. Another problem, that may be severely increased in CRPs that involve large groups, is the necessity of finding and dealing with experts and subgroups who do not cooperate to achieve an agreement, because they are not prone to modify their initial position or even they move their preferences against the collective opinion. Detecting and managing such behaviors becomes then crucial to reach an agreement more efficiently [3].

This contribution presents an approach based on fuzzy clustering techniques to detect and deal with non-cooperative behaviors in large-scale CRPs. Such an approach is integrated into a consensus model whose design allows a high degree of automation and its implementation into a Consensus Support System, which may facilitate the management of large groups of experts.

This paper is organized as follows: Section 2 reviews some preliminaries related to CRPs in GDM and fuzzy clustering. In Section 3, a consensus model that incorporates the methodology to detect and manage experts' non-cooperative behaviors is presented. Section 4 shows an illustrative example of the proposal's performance, and Section 5 concludes the paper.

\section{Preliminaries}

This section reviews some preliminary concepts about consensus reaching in GDM and fuzzy clustering techniques.

\subsection{Consensus Reaching in Group Decision Making}

GDM problems [1] are defined as decision situations where a set $E=\left\{e_{1}, \ldots, e_{m}\right\},(m \geq 2)$, of decision makers or experts express their preferences over a finite set of alternatives $X=\left\{x_{1}, \ldots, x_{n}\right\},(n \geq 2)$ by using 
a preference structure, for instance a fuzzy preference relation:

$$
P_{i}=\left(\begin{array}{llc}
- & \ldots & p_{i}^{1 n} \\
\vdots & \ddots & \vdots \\
p_{i}^{n 1} & \ldots & -
\end{array}\right)
$$

where each assessment $p_{i}^{l k}=\mu_{P_{i}}\left(x_{l}, x_{k}\right) \in[0,1]$ represents $e_{i}$ 's preference degree of alternative $x_{l}$ over $x_{k}$, $(l \neq k)[9]$.

It is usual in many GDM situations that each expert $e_{i} \in E$ has associated an importance weight $w_{i} \in[0,1]$, due to different reasons, such as the existence of experts with different degree of expertise or knowledge about the problem [10]. The specific meaning and use of such weights in the consensus model presented in this paper, will be illustrated in Sect. 3 .

The resolution of GDM problems by applying a selection process solely $[2,11]$ does not always guarantee a decision that is accepted by all experts in the group, since some of them might consider that their opinions have not been sufficiently considered. CRPs as part of the decision process have attained a great attention to overcome this limitation [3]. In a CRP, experts modify their initial opinions, making them closer to each other and towards a collective opinion which must be satisfactory for all of them.

Different approaches for consensus have been proposed in the literature, for example the so-called notion of soft consensus, proposed by J. Kacprzyk in [1] and based on the concept of fuzzy linguistic majority, which establishes that consensus exists if most experts, participating in a problem, agree on the most important alternatives.

Classically, the process to reach a consensus is a dynamic and iterative discussion process coordinated by a human moderator, who is responsible for guiding experts in the overall process [3]. A basic scheme of the phases required for conducting CRPs is briefly described below (see Fig. 1):

1) Gathering Preferences: Each expert provides his/her preferences on the existing alternatives to the moderator.

2) Determine Degree of Consensus: The moderator computes the level of agreement in the group by means of a consensus measure, based on similarity measures and aggregation operators.

3) Consensus Control: The consensus degree is compared with a threshold level of agreement desired by the group. If such degree is enough, the group moves on to the selection process, otherwise, more discussion rounds are required.

4) Generate Feedback Information: The moderator advises experts, suggesting them how to modify their opinions to make them closer and increase the level of agreement in the group. Afterwards, a new discussion round begins.

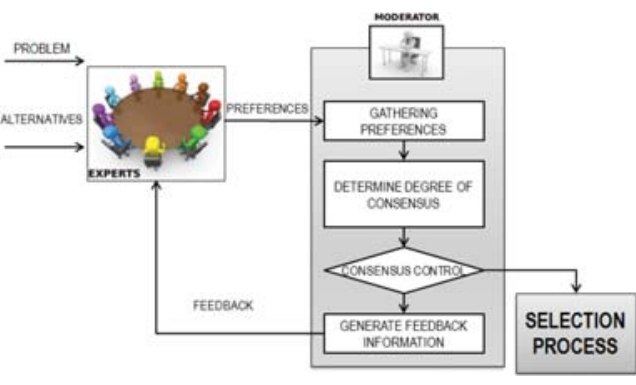

Fig. 1: General scheme for CRPs.

\subsection{Fuzzy Clustering}

The method proposed in this paper to detect noncooperative experts' behaviors in CRPs is based on fuzzy clustering, therefore in this subsection we give a brief revision of fuzzy clustering techniques.

Clustering is a non-supervised classification technique for data analysis and interpretation [12], in which a set of data objects are separated into a number of groups so-called clusters, based on a measure of similarity, so that data objects within the same cluster are more similar to each other than data objects belonging to different clusters [13]. Usually, each cluster is represented by a prototype or cluster centre that characterizes all data objects in the cluster.

Although traditional clustering methods assume that each data object can belong to one cluster only, fuzzy clustering-based approaches, where data objects may belong to multiple clusters with different degrees of membership, have also arisen $[12,13]$. Such methods seek to find cluster centres for a predefined number $N$ of clusters and assign each data object a fuzzy membership degree to each cluster. One of the most extended fuzzy clustering algorithms is Fuzzy C-Means (FCM) [14]. In FCM, cluster centres and data objects are iteratively updated until a locally optimal solution, determined by a pre-established stopping condition, is found.

Some necessary assumptions for adapting FCM to our proposal for GDM based on fuzzy preference relations, are:

- A data object is an expert's fuzzy preference relation $P_{i}$, hence cluster centres $C_{h}$ are also structured as fuzzy preference relations.

- A parameter $b>1$ indicates fuzziness degree of clusters. The larger $b$, the fuzzier the clusters are [14].

- Membership degrees to each cluster, $\mu_{C_{h}}\left(P_{i}\right)$, are computed by using distance metrics. Let $d$ denote a distance metric (e.g. based on Minkowski distance). Then, $d\left(P_{i}, C_{h}\right)$ represents the distance between $P_{i}$ and a cluster centre $C_{h}$ (see Sect. 3.2 for more detail about its computation). 


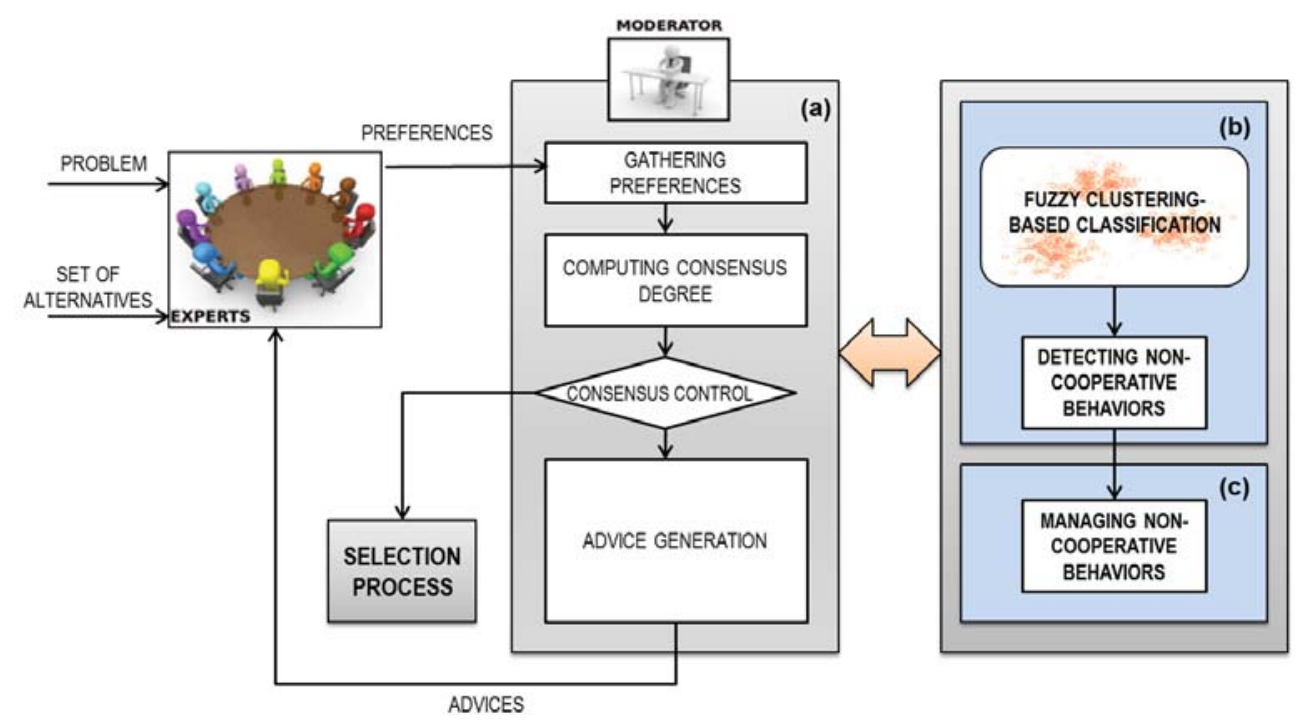

Fig. 2: Consensus model scheme.

A description of the FCM algorithm on a set $\left\{P_{1}, \ldots, P_{m}\right\}$ of fuzzy preference relations is shown below:

1. Set number of clusters $N \geq 2$ and fuzziness degree b.

2. Initialize $N$ clusters $C_{h}, h=1, \ldots, N$, by means of a cluster initialization technique [15].

3. Compute membership degrees of each preference relation $P_{i}$ to each cluster $C_{h}, \mu_{C_{h}}\left(P_{i}\right) \in[0,1]$ :

$$
\mu_{C_{h}}\left(P_{i}\right)=\frac{\left(1 / d\left(P_{i}, C_{h}\right)\right)^{1 /(b-1)}}{\sum_{u=1}^{N}\left(1 / d\left(P_{i}, C_{u}\right)\right)^{1 /(b-1)}}
$$

4. Update cluster centres:

$$
C_{h}=\frac{\sum_{i=1}^{m} \mu_{C_{h}}\left(P_{i}\right) P_{i}}{\sum_{i=1}^{m} \mu_{C_{h}}\left(P_{i}\right)}
$$

5. Repeat steps 3,4 until a stopping condition is reached. Further detail about the stopping condition considered in this proposal can be found in Sect. 3.2.

\section{Consensus Model for Detecting Non-Cooperative Behaviors}

This section presents a novel consensus model for large-scale GDM problems. Its main novelty is the fuzzy clustering-based approach it integrates to classify experts, based on their preference values, in order to detect non-cooperative behaviors across the CRP and manage them.

Figure 2 shows a scheme of the model, which is divided into three components: (a) A general scheme of the model that supports the main phases usually conducted in CRPs (see Sec. 2.1).

(b) A fuzzy clustering-based method to classify experts' preferences and a rule-based approach for non-cooperative behavior detection.

(c) A method based on updating experts' weights $w_{i}$ to manage non cooperating experts previously detected.

Such components are developed in detail in the following subsections.

\subsection{Consensus Model Scheme}

The proposed consensus model extends some ideas from $[4,5]$. Each $e_{i} \in E$ is given an importance weight $w_{i} \in[0,1]$, which is maximum for all experts in the group at the beginning of the CRP, i.e. $w_{i}=1$, $\forall i \in\{1, \ldots, m\}$. The values of such weights may vary during the discussion process as a result of detecting non-cooperative behaviors within the group (as will be shown in Sect. 3.3).

The main phases of the model are described below. As will be pointed out during the description of the model, detection and management of noncooperative behaviors takes place at some steps across these phases:

(1) Gathering Preferences.

Each $e_{i}$ provides a fuzzy preference relation $P_{i}=$ $\left(p_{i}^{l k}\right)_{n \times n}$ on alternatives in $X$.

(2) Computing Consensus Degree.

The level of agreement amongst experts is computed, by means of the following steps:

1. For each pair of experts $e_{i}, e_{j},(i<j)$ a similarity matrix $S M_{i j}=\left(s m_{i j}^{l k}\right)^{n \times n}$ is computed, being $s m_{i j}^{l k} \in[0,1]$ the degree of similarity between $p_{i}^{l k}$ 
and $p_{j}^{l k}$, obtained as follows:

$$
s m_{i j}^{l k}=1-\left|\left(p_{i}^{l k}-p_{j}^{l k}\right)\right|
$$

2. A consensus matrix $C M=\left(\mathrm{cm}^{l k}\right)_{n \times n}$, is computed by aggregating similarity matrices, taking into account experts' importance weights. Each element $c m^{l k} \in[0,1], l \neq k$, is computed as:

$$
c m^{l k}=\frac{\sum_{i=1}^{m-1} \sum_{j=i+1}^{m} w_{i j} s m_{i j}^{l k}}{\sum_{i=1}^{m-1} \sum_{j=i+1}^{m} w_{i j}}
$$

where $w_{i j}$ is the weight associated to the pair $\left(e_{i}, e_{j}\right)$, as will be explained in Sect. 3.3. $\mathrm{cm}^{l k}$ represents the consensus degree on the pair of alternatives $\left(x_{l}, x_{k}\right), l, k \in\{1, \ldots, n\}, l \neq k$.

3. The degree of consensus on each alternative $x_{l} \in$ $X$ is computed as:

$$
c a^{l}=\frac{\sum_{k=1, k \neq l}^{n} c m^{l k}}{n-1}
$$

4. Finally, a global consensus degree is computed:

$$
c r=\frac{\sum_{l=1}^{n} c a^{l}}{n}
$$

\section{(3) Consensus Control.}

The global consensus degree $c r$ is compared with a consensus threshold $\mu \in[0,1]$ established a priori by the group. If $c r \geq \mu$, then the CRP ends; otherwise, another discussion round is required. A parameter Maxround can be used to limit the number of rounds if consensus is not achieved.

\section{(4) Advice Generation.}

When the degree of consensus is not sufficient, i.e. $\mathrm{cr}<\mu$, experts must modify their preferences in order to make them closer to each other in subsequent rounds of discussion. Hence, this phase is introduced in the consensus model to automate the tasks carried out by the human moderator to identify the furthest experts' preferences from consensus, and generate the necessary rules to advise them on how to change such preferences to increase the level of agreement in the group. The following steps are considered in the advice generation phase:

1. A collective preference $P_{c}=\left(p_{c}^{l k}\right)_{n \times n}$ is computed by aggregating preference relations:

$$
p_{c}^{l k}=\frac{\sum_{i=1}^{m} w_{i} p_{i}^{l k}}{\sum_{i=1}^{m} w_{i}}
$$

being $w_{i} \in[0,1]$ the importance weight of $e_{i}$. Again, weight-based management of noncooperative behaviors (see Sect. 3.3), directly affects computations at this step.
2. Once computed $P_{c}$, the fuzzy clustering-based approach to manage non-cooperative behaviors in the current round, and the subsequent management of such behaviors, are applied (see Sects. 3.2 and 3.3). The results of applying these processes affect computations shown in Eqs. (4) and (7), in the following round of the CRP.

3. Proximity matrices $P P_{i}=\left(p p_{i}^{l k}\right)_{n \times n}$ indicating the closeness between $P_{i}$ and $P_{c}$, are computed:

$$
p p_{i}^{l k}=1-\left|\left(p_{i}^{l k}-p_{c}^{l k}\right)\right|
$$

Proximity values are used to identify the furthest preferences from $P_{c}$ that should be modified by some experts.

4. Pairs of alternatives $\left(x_{l}, x_{k}\right)$ whose consensus degrees $c a^{l}$ and $c p^{l k}$ are not enough, are identified:

$$
C C=\left\{\left(x_{l}, x_{k}\right) \mid c a^{l}<c r \wedge c p^{l k}<c r\right\}
$$

Based on $C C$, experts who should change their opinion on a pair $\left(x_{l}, x_{k}\right) \in C C$ are identified. An average proximity $\overline{p p}^{l k}$, given by:

$$
\overline{p p}^{l k}=\frac{\sum_{i=1}^{m} p p_{i}^{l k}}{m}
$$

is used to identify them, so that those $e_{i}$ s whose $p p_{i}^{l k}<\overline{p p}^{l k}$ are advised to modify their assessment on pair $\left(x_{l}, x_{k}\right)$.

5. Establish change directions: Several direction rules are applied to suggest the direction of changes on experts' assessments, in order to increase the level of agreement in the following rounds. An acceptability threshold $\varepsilon \geq 0$ can be used to allow a margin of acceptability when $p_{i}^{l k}$ and $p_{c}^{l k}$ are close enough to each other.

- DIR.1: If $\left(p_{i}^{l k}-p_{c}^{l k}\right)<-\varepsilon$, then $e_{i}$ should increase his/her assessment $p_{i}^{l k}$.

- DIR.2: If $\left(p_{i}^{l k}-p_{c}^{l k}\right)>\varepsilon$, then $e_{i}$ should decrease his/her assessment $p_{i}^{l k}$.

- DIR.3: If $-\varepsilon \leq\left(p_{i}^{l k}-p_{c}^{l k}\right) \leq \varepsilon$, then $e_{i}$ does not need to modify his/her assessment $p_{i}^{l k}$.

\subsection{Fuzzy Clustering-based Behavior Detection}

Here, we present the second component of the consensus model proposed in this paper (see Fig. 2(b)): a method to classify experts' preferences and detect subgroups of them who present a behavior contrary to consensus achievement during the CRP. The aim of such a method is to deal with non cooperating experts detected appropriately (see Sect. 3.3), in order to improve the overall CRP performance.

The method is applied once at each CRP round during the Advice Generation phase (see Sect. 3.1). Let $t \in\{1, \ldots$, Maxround -1$\}$ be the current round of the 
CRP. Then, $e_{i}$ 's preference in round $t$ will be denoted as $P_{i}^{t}$, and each cluster centre in such a round will be denoted as $C_{h}^{t}, h=1, \ldots, N$.

The proposed detection method is organized into two phases, they are described in detail below.

\subsubsection{FCM-based classification}

Firstly, a clustering technique based on FCM to classify experts according to their preferences is applied, considering the following specifications:

- A fuzziness degree $b=2$ is taken.

- $N$ clusters are initialized according to the method proposed in [15], and choosing $P_{c}^{t}$ as the centre of the first cluster, $C_{1}^{t}$.

- Cluster centres $C_{h}^{t}(h \geq 2)$ and membership degrees $\mu_{C_{h}^{t}}\left(P_{i}^{t}\right)$ are updated iteratively, according to Eqs. (1) and (2). In order to preserve $P_{c}^{t}$ as the centre of cluster $C_{1}^{t}$ when applying detection, its values are not updated.

- Distances between preference relations and/or cluster centres are computed. To do so, here we propose computing the euclidean distance between two preference relations (resp. cluster centres), as follows:

$$
d\left(P_{i}^{t}, C_{h}^{t}\right)=\frac{\sum_{l=1}^{n} \sum_{k=1, k \neq l}^{n}\left|p_{i}^{l k, t}-c_{h}^{l k, t}\right|}{n(n-1)}
$$

- The update process ends when all clusters stabilize, i.e. the variation in membership degrees between two consecutive iterations of FCM, $y$ and $y-1$, tends to zero:

$$
\frac{\sum_{i=1}^{m} \sum_{h=1}^{N}\left|\mu_{C_{h}^{t}}^{y}\left(P_{i}^{t}\right)-\mu_{C_{h}^{t}}^{y-1}\left(P_{i}^{t}\right)\right|}{m \cdot N} \leq \epsilon
$$

being $\epsilon \approx 0$ a threshold used as stopping condition.

\subsubsection{Detection of Non-cooperative Behaviors}

Once applied the FCM-based classification algorithm, it is applied a scheme to detect non-cooperative behaviors from clusters obtained. To do so, we propose a flexible rule-based scheme, in which for each cluster $C_{h}^{t}$ $(h \geq 2)$, a set of rules are checked to decide about the existence of a non-cooperative behavior. If all rules are accomplished by such a cluster, then some experts belonging to it present a non-cooperative behavior during the CRP. Formally, let $R_{1} \ldots R_{q}$ be a set of rules indicating a condition or property of a cluster $C_{h}^{t}$, which may be either true or false. Then, we propose verifying the following proposition: "IF $R_{1} \wedge \ldots \wedge R_{q} T H E N$ Non-cooperative behavior detected".
The general rule-based approach proposed is flexible, in the sense that rules can be added, modified and/or removed from the consensus model, according to the specific GDM problem to solve and the needs of the group. Nevertheless, in this paper such an approach will be instantiated, by defining some specific rules in order to illustrate their use. The current scheme will be separated into two phases (see Fig. 3 ): one for detecting subgroup behaviors and another one for detecting non cooperating experts within the subgroup detected. The detection rules presented below are first applied in the second round of the CRP, i.e. when $t=2$, because comparisons with clusters in round $t-1$ are required.

A non cooperating subgroup is detected in a cluster $C_{h}^{t}(h \geq 2)$, if it accomplishes rules $\mathrm{S} 1$ and S2 (see Fig. 3):

S1. Similar Cluster Composition: This rule determines whether a cluster is compound by the same experts across the time. Similarities between a given cluster $C_{h}^{t}(h \geq 2)$ and each cluster in the previous round $C_{u}^{t-1}(u=2, \ldots, N)$, are computed:

$$
\operatorname{sim}\left(C_{h}^{t}, C_{u}^{t-1}\right)=1-\frac{\sum_{i=1}^{m} \Delta_{h u}^{t}\left(P_{i}\right)}{m}
$$

being $\Delta_{h u}^{t}\left(P_{i}\right) \in[0,1]$ the variation in $P_{i}$ membership to both clusters across time, computed as:

$$
\Delta_{h u}^{t}\left(P_{i}\right)=\left|\mu_{C_{h}}^{t}\left(P_{i}^{t}\right)-\mu_{C_{u}}^{t-1}\left(P_{i}^{t-1}\right)\right|
$$

A similarity threshold $\kappa \in[0,1]$, whose value should be close to 1 , is defined to decide whether cluster similarity is enough to accomplish this rule: if $\exists C_{u}^{t-1}: \operatorname{sim}\left(C_{h}^{t}, C_{u}^{t-1}\right) \geq \kappa$, then $C_{h}^{t}$ and $C_{u}^{t-1}$ are assumed to represent the same cluster across the time.

S2. Further Distance to $P_{c}$ : Assume that, based on rule $\mathrm{S} 1, C_{h}^{t}$ and $C_{u}^{t-1}$ are considered the same cluster. Distances between $C_{h}$ and the collective preference, $P_{c} \equiv C_{1}$, in rounds $t$ and $t-1$, are computed as $d\left(C_{1}^{t}, C_{h}^{t}\right)$ and $d\left(C_{1}^{t-1}, C_{u}^{t-1}\right)$ respectively, by means of Eq. (11). Let $\nu \in[0,1]$ be a threshold so-called minimum distance to analyze subgroup behaviors, such that if a distance between two different clusters in round $t$ is lower than $\nu$, then their cluster centres are close enough to consider that they represent similar opinions. $\nu$ should take a low value, normally not higher than 0.1. If $d\left(C_{1}^{t}, C_{h}^{t}\right)>\nu$ AND $d\left(C_{1}^{t}, C_{h}^{t}\right) \geq$ $d\left(C_{1}^{t-1}, C_{u}^{t-1}\right)$, then some experts in cluster $C_{h}$ are moving further from the collective opinion $P_{c}$.

Once detected a subgroup non-cooperative behavior, it is necessary to identify those experts in the 


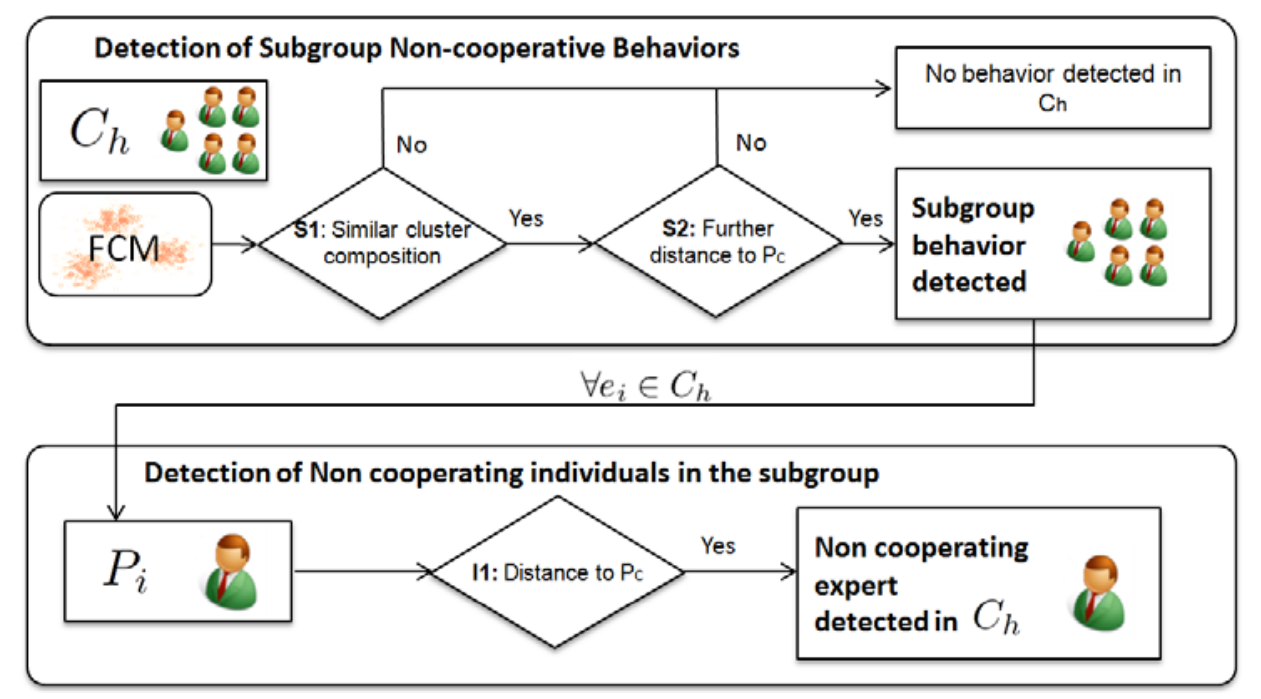

Fig. 3: Rule-based scheme for the detection of non-cooperative behaviors.

subgroup who present such behavior, in order to deal with them. Consider that $e_{i} \in C_{h}^{t}$ iff $\mu_{C_{h}^{t}}\left(P_{i}^{t}\right)=$ $\max _{u} \mu_{C_{u}^{t}}\left(P_{i}^{t}\right), u \in\{1, \ldots, N\}$. Then, for each $e_{i} \in$ $C_{h}^{t}$, the following rule is checked to decide whether he/she takes part in the subgroup behavior detected (Fig. 3).

I1. Distance to $P_{c}$ : Distance between $P_{i}$ and the collective opinion increases or it is higher than the average distance to the collective opinion. This occurs if at least one of these two conditions holds:

i) $d\left(P_{i}^{t}, C_{1}^{t}\right)>\bar{d}^{t}$, where $\bar{d}^{t}$ represents the average distance to $P_{c}^{t}$, computed as:

$$
\bar{d}^{t}=\frac{\sum_{i=1}^{m} d\left(P_{i}^{t}, C_{1}^{t}\right)}{m}
$$

ii) $d\left(P_{i}^{t}, C_{1}^{t}\right)>d\left(P_{i}^{t-1}, C_{1}^{t-1}\right)$.

\subsection{Management of Behaviors Detected}

If non-cooperative behaviors are detected, then the following method is proposed to manage experts involved in them, based on updating their importance weights $w_{i}$ throughout the CRP.

Initially, $w_{i}=1, \forall i$. If a subgroup behavior is detected in a cluster $C_{h}^{t}$ in round $t \geq 2$, the procedure applied on experts' preference relations $P_{i}^{t} \in C_{h}^{t}$ that accomplished rule I1 to update their weights is as follows:

- Compute the new weight $w_{i_{n e w}}$ upon current weight $w_{i}$ :

$$
w_{i_{\text {new }}}=w_{i}\left(1-\frac{d\left(P_{i}^{t}, C_{1}^{t}\right)}{\max _{j} d\left(P_{j}^{t}, C_{1}^{t}\right)}\right)
$$

- Assign $w_{i} \leftarrow w_{i_{\text {new }}}$.
Notice that $w_{i_{\text {new }}} \in\left[0, w_{i}\right] \subseteq[0,1], w_{i_{\text {new }}} \leq w_{i}$, and the higher the distance between $e_{i}$ 's preference and the collective preference, the more penalizing is applied to his/her importance weight.

As previously shown in Section 3.1, weights are considered in two steps of the CRP:

- Computing $C M$ from experts' similarities (Eq. (4)).

- Computing $P_{c}$ (Eq. (7)).

$C M$ is obtained by aggregating similarity values $s m_{i j}^{l k}$ for each pair of experts $\left(e_{i}, e_{j}\right)$, therefore it is necessary to combine $w_{i}$ and $w_{j}$ to obtain a weight $w_{i j}$ associated to such a pair. Assuming that, if at least one expert in the pair $\left(e_{i}, e_{j}\right)$ presents a behavior contrary to consensus achievement then the importance weight $w_{i j}$ assigned to $s m_{i j}^{l k}$ should be low, we propose computing it as $w_{i j}=\min \left(w_{i}, w_{j}\right)$.

\section{Illustrative Example}

This section illustrates the use of an implemented version of the consensus model presented, in the resolution of a real-life large-scale GDM problem, where some experts might present non-cooperative behaviors during the CRP. The aim of such an example is to prove the effectiveness of the approach presented in this paper for fuzzy clustering-based behavior detection.

The problem is formulated as follows: a group of 41 students from Computer Science M.Sc. Degree, $E=\left\{e_{1}, \ldots, e_{41}\right\}$, must make an agreed decision about choosing a place to celebrate their graduation dinner in Jaén (Spain). The set of proposed restaurants $X=\left\{x_{1}, x_{2}, x_{3}, x_{4}\right\}$ is:

- $x_{1}$ : 'Santa Catalina' castle.

- $x_{2}$ : 'Los Caballos' ranch. 
Table 1: Parameters considered for consensus reaching and detection processes.

\begin{tabular}{|l|l|}
\hline Parameter description & Value \\
\hline Consensus threshold & $\mu=0.85$ \\
\hline Maximum number of rounds & Maxround $=10$ \\
\hline Acceptability threshold & $\varepsilon=0.02$ \\
\hline Number of clusters & $N=4$ \\
\hline Fuzziness degree in FCM & $b=2$ \\
\hline Stopping condition threshold in FCM & $\epsilon=0.001$ \\
\hline Minkowski distance chosen & $p=1$ \\
\hline Cluster similarity threshold (rule S1) & $\kappa=0.9$ \\
\hline Minimum distance to analyze group behaviors (rule S2) & $\nu=0.05$ \\
\hline
\end{tabular}

Table 2: Consensus degree $\mathrm{cr}$ achieved and detection of non-cooperative behaviors in each CRP round.

\begin{tabular}{|c||c||c|c|}
\hline \multicolumn{1}{|c||}{$t$} & Without Detection & \multicolumn{2}{c|}{ Non-coop. Behavior Detection } \\
\hline & cr & cr & Subgroup detected \\
\hline 1 & 0.63060 & 0.63060 & - \\
\hline 2 & 0.66655 & 0.66614 & - \\
\hline 3 & 0.69543 & 0.69473 & $\sqrt{ }$ \\
\hline 4 & 0.71914 & 0.74663 & $\sqrt{ }$ \\
\hline 5 & 0.72266 & 0.84651 & $\sqrt{ }$ \\
\hline 6 & 0.73317 & $\mathbf{0 . 8 5 7 4 3}$ & \\
\hline 7 & 0.73525 & & \\
\hline 8 & 0.74312 & & \\
\hline 9 & 0.74468 & & \\
\hline 10 & 0.74427 & & \\
\hline
\end{tabular}

- $x_{3}$ : 'Pegalajar' caves.

- $x_{4}$ : 'Juleca' complex.

Table 1 summarizes parameters considered in the CRP, FCM algorithm and behavior detection mechanisms.

The model is firstly used to solve the GDM problem without applying the behavior detection and management schemes (Sects. 3.2 and 3.3). Then, the problem is solved again by applying such schemes. Our hypothesis states that applying a process to detect and manage non-cooperative behaviors might improve the CRP performance by increasing the group convergence towards consensus and making a collective decision which is more adapted to those experts who contributed to achieve an agreement.

Once conducted the CRP, results are shown and analyzed. Table 2 shows the evolution of the consensus degree $c r$ achieved in both experiments, as well as behaviors detected when applying the proposed methodology. When no behavior detection and treatment is applied, consensus is not achieved, presumably due to the existence of non cooperating subgroups of experts. Such behaviors are actually detected when the approach is applied: a subgroup behavior is first detected in the third round, therefore importance weights of experts involved in such a behavior are updated corre- spondingly, which causes two beneficial effects in the CRP:

- The convergence of $\mathrm{cr}$ towards consensus improves from the fourth round onwards, due to the effect of updated weights in the computation of $C M$.

- The collective preference becomes closer to experts who cooperate to achieve an agreement, due to the effect of updated weights in the computation of $P_{c}$.

Results have shown the effectiveness and usefulness of our approach to deal with non-cooperative behaviors in CRPs with large groups.

\section{Concluding Remarks}

In this contribution, we have presented an approach, based on fuzzy clustering, to detect subgroups of experts with a behavior contrary to consensus achievement in consensus reaching processes, and manage such behaviors to improve the process performance. Such an approach has been integrated in a consensus model aimed to the resolution of large-scale group decision making problems, which are increasingly necessary nowadays in emerging contexts, such as social networks and e-democracy. 


\section{Acknowledgment}

This work is partially supported by the Research Project TIN-2012-31263 and ERDF.

\section{References}

[1] J. Kacprzyk. Group decision making with a fuzzy linguistic majority. Fuzzy Sets and Systems, 18(2):105-118, 1986.

[2] F. Herrera, E. Herrera-Viedma, and J. Verdegay. A sequential selection process in group decision making with linguistic assessments. Information Sciences, 85(1995):223-239, 1995.

[3] S. Saint and J. R. Lawson. Rules for Reaching Consensus. A Modern Approach to Decision Making. Jossey-Bass, 1994.

[4] F. Mata, L. Martínez, and E. Herrera-Viedma. An adaptive consensus support model for group decision-making problems in a multigranular fuzzy linguistic context. IEEE Transactions on Fuzzy Systems, 17(2):279-290, 2009.

[5] I. Palomares, J. Liu, Y. Xu, and L. Martínez. Modelling experts' attitudes in group decision making. Soft Computing, 16(10):1755-1766, 2012.

[6] R.O. Parreiras, P. Ekel, J.S.C. Martini, and R.M. Palhares. A flexible consensus scheme for multicriteria group decision making under linguistic assessments. Information Sciences, 180(7):10751089, 2010.

[7] J. Kim. A model and case for supporting participatory public decision making in e-democracy. Group Decision and Negotiation, 17(3):179-192, 2008.

[8] C. Sueur, J.L. Deneubourg, and O. Petit. From social network (centralized vs. decentralized) to collective decision-making (unshared vs. shared consensus). PLoS one, 7(2):1-10, 2012.

[9] S.A. Orlovsky. Decision-making with a fuzzy preference relation. Fuzzy Sets and Systems, $1(3): 155-167,1978$.

[10] J. Lu, G. Zhang, D. Ruan, and F. Wu. MultiObjective Group Decision Making. Imperial College Press, 2006.

[11] M. Roubens. Fuzzy sets and decision analysis. Fuzzy Sets and Systems, 90(2):199-206, 1997.

[12] W. Pedrycz. Knowledge-based Clustering, chapter Clustering and Fuzzy Clustering, pages 1-27. Wiley, 2005.

[13] V. Cherkassky and F. Mulier. Learning from Data: Concepts, Theory and Methods. Wiley, 2007.

[14] J. Bezdek. Pattern Recognition with Fuzzy Objective Function Algorithms. Wiley, 2007.

[15] I. Katsavounidis, C.C. Jay-Kuo, and Z. Zhang. A new initialization technique for generalized lloyd iteration. IEEE Signal Processing Letters, 1(10):144-146, 1994. 